Special issue of the 3rd International Conference on Computational and Experimental Science and Engineering (ICCESEN 2016)

\title{
Laguerre Polynomial Solutions of a Class of Delay Partial Functional Differential Equations
}

\author{
B. GÜrbüZ* AND M. SEZER
}

Manisa Celal Bayar University, Department of Mathematics, Faculty of Art and Science, Manisa, Turkey

In this study, we develop a novel matrix collocation method based on the Laguerre polynomials to find the approximate solutions of some parabolic delay differential equations with integral terms subject to appropriate initial and boundary conditions. The method reduces the solution of the mentioned equations to the solution of a matrix equation which corresponds to system of algebraic equations with unknown Laguerre coefficients. Besides, the error analysis together with numerical results are performed to illustrate the efficiency of our method computationally.

DOI: 10.12693/APhysPolA.132.558

PACS/topics: Laguerre polynomials and series, delay partial differential equations with integral terms, matrix and collocation methods, error estimations

\section{Introduction}

Delay partial and partial integro-differential equations play an important role in many scientific areas such as biology, physics, and engineering [1-7]. Furthermore, the approximation methods of these type of problems have been developed by many authors [8-12]. Particularly, we represent a novel approximation to solve a class of delay partial functional differential equations with integral terms in the form

$$
\begin{aligned}
& u_{t}(x, t)=u_{x x}(x, t)+g(x, t)+a(x, t) u(x, \alpha t) \\
& \quad+\int_{0}^{t} K(x, t, s) u(x, s) \mathrm{d} s,
\end{aligned}
$$

under the initial and boundary conditions

$$
\begin{aligned}
& u(x, 0)=f(x), u_{t}(x, 0)=m(x), u(0, t)=h(t), \\
& u(l, t)=n(t), 0 \leq x \leq l, 0 \leq t \leq T .
\end{aligned}
$$

The functions $g, a, f, m, h$, and $n$ are continuous functions; $\alpha$ is the arbitrary constant; and $K$ is the kernel function of the integral part of (1) on $R^{2}$. We assume the approximate solution of the problem (1)-(2) in the truncated Laguerre series form

$$
\begin{aligned}
& u(x, t) \cong \sum_{n=0}^{N} \sum_{p=0}^{N} a_{n, p} L_{n, p}(x, t), \\
& L_{n, p}(x, t)=L_{n}(x) L_{p}(t),
\end{aligned}
$$

where $L_{n}(x)$ denotes the Laguerre polynomials

$$
L_{n}(x)=\sum_{k=0}^{n} \frac{(-1)^{k}}{k !}\left(\begin{array}{l}
n \\
k
\end{array}\right) x^{k}, n \in N, 0 \leq x<\infty
$$

and $a_{n, p},(n, p=0,1, \ldots, N)$ are unknown Laguerre polynomial coefficients, and $N$ is chosen as any positive integer such that $L_{0}(x)=1, L_{1}(x)=1-x, N \geq 2$.

\footnotetext{
*corresponding author; e-mail: burcu.gurbuz@cbu.edu.tr
}

\section{Fundamental relations and Laguerre collocation method}

In this section, we present an approximate solution in terms of the Laguerre polynomials in the form (3). First we can write (3) in the matrix form

$$
\begin{aligned}
& {[u(x, t)]=\boldsymbol{L}(x) \overline{\boldsymbol{L}}(t) \boldsymbol{A} ;} \\
& \boldsymbol{L}(x)=\left[\begin{array}{llll}
L_{0}(x) & L_{1}(x) & \ldots & L_{N}(x)
\end{array}\right], \\
& \overline{\boldsymbol{L}}(t)=\operatorname{diag}(\boldsymbol{L}(t), \boldsymbol{L}(t), \ldots, \boldsymbol{L}(t)), \\
& \boldsymbol{A}=\left[\begin{array}{llll}
a_{i 0} & a_{i 1} & \ldots & a_{i N}
\end{array}\right]^{T},
\end{aligned}
$$

where $i=0,1, \ldots, N$, then, we use the matrix relation

$$
\boldsymbol{L}(x)=\boldsymbol{X}(x) \boldsymbol{H} \text { and } \overline{\boldsymbol{L}}(t)=\overline{\boldsymbol{X}}(t) \overline{\boldsymbol{H}}
$$

where $\boldsymbol{X}(x)=\left[\begin{array}{llll}1 & x & \ldots & x^{N}\end{array}\right]$;

$$
\begin{aligned}
& \overline{\boldsymbol{X}}(t)=\operatorname{diag}(\boldsymbol{X}(t) \boldsymbol{X}(t), \ldots, \boldsymbol{X}(t)), \\
& \boldsymbol{H}=
\end{aligned}
$$

$$
\begin{gathered}
{\left[\begin{array}{ccccc}
\frac{(-1)^{0}}{0 !}\left(\begin{array}{l}
0 \\
0
\end{array}\right) & \frac{(-1)^{0}}{0 !}\left(\begin{array}{l}
1 \\
0
\end{array}\right) & \frac{(-1)^{0}}{0 !}\left(\begin{array}{l}
2 \\
0
\end{array}\right) & \cdots & \frac{(-1)^{0}}{0 !}\left(\begin{array}{c}
N \\
0
\end{array}\right) \\
0 & \frac{(-1)^{1}}{1 !}\left(\begin{array}{l}
1 \\
1
\end{array}\right) & \frac{(-1)^{1}}{1 !}\left(\begin{array}{l}
2 \\
1
\end{array}\right) & \cdots & \frac{(-1)^{1}}{1 !}\left(\begin{array}{c}
N \\
1
\end{array}\right) \\
0 & 0 & \frac{(-1)^{2}}{2 !}\left(\begin{array}{l}
2 \\
2
\end{array}\right) & \ldots & \frac{(-1)^{2}}{2 !}\left(\begin{array}{c}
N \\
2
\end{array}\right) \\
\vdots & \vdots & \ddots & \vdots \\
0 & 0 & 0 & \cdots & \frac{(-1)^{N}}{N !}\left(\begin{array}{c}
N \\
N
\end{array}\right)
\end{array}\right],} \\
\overline{\boldsymbol{H}}=\operatorname{diag}(\boldsymbol{H}, \boldsymbol{H}, \ldots, \boldsymbol{H}) \\
{[u(x \alpha t)]=\boldsymbol{L}(x) \overline{\boldsymbol{L}}(\alpha t) \boldsymbol{A},} \\
\overline{\boldsymbol{L}}(\alpha t)=\overline{\boldsymbol{X}}(\alpha t) \overline{\boldsymbol{H}}=\overline{\boldsymbol{X}}(t) \overline{\boldsymbol{B}}(\alpha, 0) \overline{\boldsymbol{H}} .
\end{gathered}
$$

Moreover, the matrices $\boldsymbol{X}(x), \overline{\boldsymbol{X}}(t)$ and their derivatives $\boldsymbol{X}^{\prime}(x), \boldsymbol{X}^{\prime \prime}(x)$ and $\overline{\boldsymbol{X}}^{\prime}(t), \overline{\boldsymbol{X}}^{\prime \prime}(t)$ can be related as $\boldsymbol{X}^{\prime}(x)=\boldsymbol{X}(x) \boldsymbol{B}, \boldsymbol{X}^{\prime \prime}(x)=\boldsymbol{X}(x) \boldsymbol{B}^{2}, \overline{\boldsymbol{X}}^{\prime}(t)=\overline{\boldsymbol{X}}(t) \overline{\boldsymbol{B}}$, 


$$
\begin{aligned}
& \overline{\boldsymbol{X}}^{\prime \prime}(t)=\overline{\boldsymbol{X}}(t) \overline{\boldsymbol{B}}^{2} ; \\
& \boldsymbol{B}=\left[\begin{array}{lllll}
0 & 1 & 0 & \ldots & 0 \\
0 & 0 & 2 & \ldots & 0 \\
\vdots & \vdots & \vdots & \ddots & \vdots \\
0 & 0 & 0 & N & 0 \\
0 & 0 & 0 & 0 & 0
\end{array}\right], \\
& \overline{\boldsymbol{B}}(\alpha, 0)=\operatorname{diag}(\boldsymbol{B}(\alpha, 0), \boldsymbol{B}(\alpha, 0), \ldots, \boldsymbol{B}(\alpha, 0)), \\
& \boldsymbol{B}(\alpha, 0)=\operatorname{diag}\left((\alpha)^{0},(\alpha)^{1}, \ldots,(\alpha)^{N}\right) .
\end{aligned}
$$

Then, we organize the matrix relations of derivative forms of $u(x, t)$ with $(5),(6)$ and $(8)$

$$
\begin{aligned}
& {\left[u_{x}(x, t)\right]=\boldsymbol{L}^{\prime}(x) \overline{\boldsymbol{L}}(t) \boldsymbol{A}=\boldsymbol{X}(x) \boldsymbol{B} \boldsymbol{H} \overline{\boldsymbol{L}}(t) \boldsymbol{A},} \\
& {\left[u_{x x}(x, t)\right]=\boldsymbol{L}^{\prime \prime}(x) \overline{\boldsymbol{L}}(t) \boldsymbol{A}=\boldsymbol{X}(x) \boldsymbol{B}^{2} \boldsymbol{H} \overline{\boldsymbol{L}}(t) \boldsymbol{A},} \\
& {\left[u_{t}(x, t)\right]=\boldsymbol{L}(x) \overline{\boldsymbol{L}}^{\prime}(t) \boldsymbol{A}=\boldsymbol{X}(x) \boldsymbol{H} \overline{\boldsymbol{L}}^{\prime}(t) \boldsymbol{A} .}
\end{aligned}
$$

On the other hand, the function $K(x, t, s)$ can be expressed by the truncated Taylor series

$$
\begin{aligned}
& K(x, t, s)=\sum_{p=0}^{N} \sum_{q=0}^{N} \sum_{r=0}^{N} k_{p q r} x^{p} t^{q} s^{r}, \\
& k_{p q r}=\frac{1}{p ! q ! r !} \frac{\partial^{p+q+r} K(0,0,0)}{\partial x^{p} \partial t^{q} \partial s^{r}}, K=\left[k_{p q r}\right], \\
& p, q, r=0,1, \ldots, N
\end{aligned}
$$

and the matrix forms of $u(x, s)$ and $K(x, t, s)$ become

$$
\begin{aligned}
& {[u(x, s)]=\boldsymbol{X}(x) \boldsymbol{H} \overline{\boldsymbol{X}}(s) \overline{\boldsymbol{H}} \boldsymbol{A}=\boldsymbol{X}(s) \boldsymbol{H} \overline{\boldsymbol{X}}(x) \overline{\boldsymbol{H}} \boldsymbol{A}^{*},} \\
& {[K(x, t, s)]=\boldsymbol{X}(x) \overline{\boldsymbol{X}}(t) \boldsymbol{K} \boldsymbol{X}^{T}(s) .}
\end{aligned}
$$

By using (10), the matrix form of integral part is obtained as follows:

$$
\begin{aligned}
& \int_{0}^{t} K(x, t, s) u(x, s) \mathrm{d} s= \\
& \int_{0}^{t} \boldsymbol{X}(x) \overline{\boldsymbol{X}}(t) \boldsymbol{K} \boldsymbol{X}^{T}(s) \boldsymbol{X}(s) \boldsymbol{H} \overline{\boldsymbol{X}}(x) \overline{\boldsymbol{H}} \boldsymbol{A}^{*} \mathrm{~d} s= \\
& \boldsymbol{X}(x) \overline{\boldsymbol{X}}(t) \boldsymbol{K} \boldsymbol{Q}(t) \boldsymbol{H} \overline{\boldsymbol{X}}(x) \overline{\boldsymbol{H}} \boldsymbol{A}^{*}, \\
& \boldsymbol{Q}(t)=\int_{0}^{t} \boldsymbol{X}^{T}(s) \boldsymbol{X}(s) \mathrm{d} s=\left[q_{m n}(t)\right] \\
& m, n=0,1,2 \ldots, q_{m n}(t)=\frac{t^{m+n+1}}{m+n+1} \\
& \boldsymbol{A}_{i}^{*}=\left[\begin{array}{llll}
a_{0 i} & a_{1 i} & \ldots & a_{N i}
\end{array}\right]^{T}, \\
& \boldsymbol{A}^{*}=\left[\begin{array}{llll}
\boldsymbol{A}_{0}^{*} & \boldsymbol{A}_{1}^{*} & \ldots & \boldsymbol{A}_{N}^{*}
\end{array}\right]^{T} .
\end{aligned}
$$

By substituting the relations (9) and (11) into Eq. (1), we have the matrix form of $(1)$ :

$$
\begin{aligned}
& \left\{\boldsymbol{X}(x) \boldsymbol{H} \overline{\boldsymbol{L}}^{\prime}(t)-\boldsymbol{X}(x) \boldsymbol{B}^{2} \boldsymbol{H} \overline{\boldsymbol{L}}(t)\right. \\
& \quad-[a(x, t)] \overline{\boldsymbol{X}}(t) \overline{\boldsymbol{B}}(\alpha, 0) \overline{\boldsymbol{H}}\} \boldsymbol{A} \\
& \quad-\{\boldsymbol{X}(x) \overline{\boldsymbol{X}}(t) \boldsymbol{K} \boldsymbol{Q}(t) \boldsymbol{H} \overline{\boldsymbol{X}}(x) \overline{\boldsymbol{H}}\} \boldsymbol{A}^{*}=[g(x, t)] .
\end{aligned}
$$

Similarly, we organize the matrix forms for the initial and boundary conditions (2) by using (9):

$$
\begin{aligned}
& {[u(x, 0)]=\boldsymbol{L}(x) \overline{\boldsymbol{L}}(0) \boldsymbol{A}=\boldsymbol{X}(x) \boldsymbol{H} \overline{\boldsymbol{L}}(0) \boldsymbol{A}, \quad[f(x)]=\lambda,} \\
& {\left[u_{t}(x, 0)\right]=\boldsymbol{L}(x) \overline{\boldsymbol{L}}^{\prime}(0) \boldsymbol{A}=\boldsymbol{X}(x) \boldsymbol{H} \overline{\boldsymbol{L}}^{\prime}(0) \boldsymbol{A},} \\
& {[m(x)]=\mu, \quad[u(0, t)]=\boldsymbol{L}(0) \overline{\boldsymbol{L}}(t) \boldsymbol{A}, \quad[h(t)]=\gamma . \quad(13)}
\end{aligned}
$$

By putting the collocation points

$$
\begin{aligned}
& x_{i}=\frac{l}{N} i, i=0,1, \ldots, N, t_{j}=\frac{T}{N} j, j=0,1, \ldots, N, \\
& h=\frac{l}{N}
\end{aligned}
$$

into Eq. (12), then we have the fundamental matrix equation as

$$
\begin{aligned}
& \left\{\boldsymbol{X}\left(x_{i}\right) \boldsymbol{H} \overline{\boldsymbol{L}}^{\prime}\left(t_{j}\right)-\boldsymbol{X}\left(x_{i}\right) \boldsymbol{B}^{2} \boldsymbol{H} \overline{\boldsymbol{L}}\left(t_{j}\right)\right. \\
& \left.-\left[a\left(x_{i}, t_{j}\right)\right] \overline{\boldsymbol{X}}\left(t_{j}\right) \overline{\boldsymbol{B}}(\alpha, 0) \overline{\boldsymbol{H}}\right\} \boldsymbol{A}-\left\{\boldsymbol{X}\left(x_{i}\right) \overline{\boldsymbol{X}}\left(t_{j}\right)\right. \\
& \left.\times \boldsymbol{K} \boldsymbol{Q}\left(t_{i}\right) \boldsymbol{H} \overline{\boldsymbol{X}}\left(x_{i}\right) \overline{\boldsymbol{H}}\right\} \boldsymbol{A}^{*}=\left[g\left(x_{i}, t_{j}\right)\right]=\boldsymbol{G} ; \\
& \boldsymbol{G}=\left[\begin{array}{llll}
\boldsymbol{G}_{1} & \boldsymbol{G}_{2} & \ldots & \boldsymbol{G}_{N}
\end{array}\right]^{T}, \boldsymbol{G}_{i}=\left[\begin{array}{llll}
g_{i 0} & g_{i 1} & \ldots & g_{i N}
\end{array}\right]^{T}
\end{aligned}
$$

and briefly

$$
\boldsymbol{W}\left(x_{i}, t_{j}\right) \boldsymbol{A}-\boldsymbol{W}^{*}\left(x_{i}, t_{j}\right) \boldsymbol{A}^{*}=\boldsymbol{G} .
$$

If we follow the same procedure for the initial and boundary conditions (13), we have

$$
\boldsymbol{U} \boldsymbol{A}=\lambda_{i}, \boldsymbol{V} \boldsymbol{A}=\mu_{i}, \boldsymbol{Z} \boldsymbol{A}=\gamma_{j}, \boldsymbol{N} \boldsymbol{A}=\eta_{j}
$$

Consequently, to obtain the solution of Eq. (1) under the conditions (2), by replacing the row matrices (16) by the last rows of the augmented matrix (15), we have the required augmented matrix. Then, Eq. (15) becomes $\left[\tilde{\boldsymbol{W}} ; \tilde{\boldsymbol{W}}^{*} ; \tilde{\boldsymbol{G}}\right]$. The augmented matrix system is solved to find the unknown Laguerre coefficients. Thus, the approximate solution $u(x, t)$ is found in the form $(3)$.

\section{Error estimation}

In this section, the error estimation for Eq. (3) is given. We define the error function for the selected points $x=$ $x_{\alpha}, t=t_{\beta} \in[l, 0] \times[0, T], \alpha, \beta=0,1, \ldots$

$$
\begin{gathered}
E_{N}\left(x_{p} t_{q}\right)=\mid u_{t}\left(x_{p}, t_{q}\right)-u_{x x}\left(x_{p}, t_{q}\right)-g\left(x_{p}, t_{q}\right) \\
-a\left(x_{p}, t_{q}\right) u\left(x_{p}, \alpha t_{q}\right)-\int_{0}^{t} K\left(x_{p}, t_{q}, s\right) u\left(x_{p}, s\right) \mathrm{d} s \mid \cong 0,
\end{gathered}
$$

where $E_{N}\left(x_{p}, t_{q}\right) \leq 10^{-k_{\alpha \beta}}=10^{-k}$ ( $k$ is positive integer $)$ and the truncation limit $N$ is increased until difference $E_{N}\left(x_{p}, t_{q}\right)$ at each of the points becomes smaller than the prescribed $10^{-k}$. On the other hand, we can use different error norms

$$
\begin{aligned}
& L_{2}-E_{N}\left(x_{\alpha}, t_{\beta}\right)=\sqrt{\sum_{i=1}^{n}\left(e_{i}\right)^{2},} \\
& L_{\infty}-E_{N}\left(x_{\alpha}, t_{\beta}\right)=\max \left(e_{i}\right), 0 \leq i \leq n, \\
& \mathrm{RMS}-E_{N}\left(x_{\alpha}, t_{\beta}\right)=\sqrt{\sum_{i=1}^{n}\left(e_{i}\right)^{2} /(n+1),}
\end{aligned}
$$

where $e_{i}=u\left(x_{i}, \tau\right)-\hat{u}\left(x_{i}, \tau\right)$; also $u$ and $\hat{u}$ are the exact and approximate solutions of the problem, respectively, and $\tau$ is an arbitrary time $t$ in $[0, T][13,14]$. 


\section{Numerical experiments}

In this section, we consider some illustrative examples to show the accuracy and efficiency of the presented method. All results have been obtained by Maple 18 and Matlab R2014b.

Example 5.1. We consider the parabolic partial integro-differential equation [15]:

$$
\frac{\partial u(x, t)}{\partial t}=\frac{\partial^{2} u(x, t)}{\partial x^{2}}+g(x, t)+\int_{0}^{t} u(x, \tau) \mathrm{d} \tau
$$

$u(x, 0)=\left(1-x^{6}\right) \sin (x), u(0, t)=u(1, t)=0,0 \leq x t \leq$ 1 , where $g(x, t)=\left(1-x^{6}\right) \cos (x)+12 x^{5} \cos (x+t)+(1+$ $\left.30 x^{4}-x^{6}\right) \sin (x+t)$ and the exact solution of the problem is $u(x, t)=\left(1-x^{6}\right) \sin (x+t)$.

We suppose that $u(x, t)$ is approximated by a truncated Laguerre series in the form $u(x, t) \cong$ $\sum_{n=0}^{N} \sum_{p=0}^{N} a_{n, p} L_{n, p}(x, t), 0 \leq x t \leq 1$. By using the procedure in Sect. 2, the fundamental matrix relations for the equation and conditions are computed and then the Laguerre coefficients are found. Thhe comparison of exact and approximate solution for $N=50$ can be seen in Fig. 1.
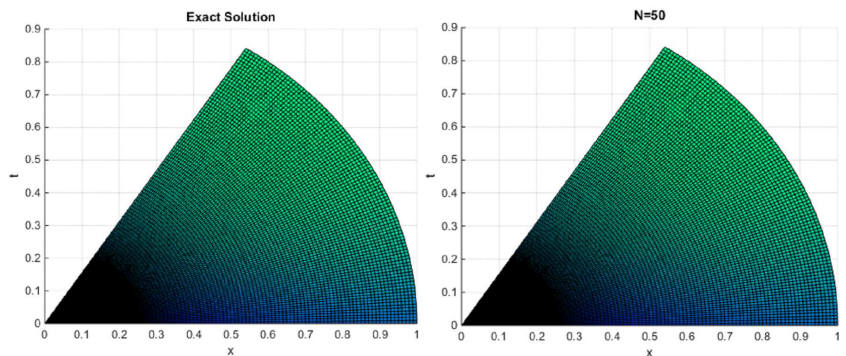

Fig. 1. Comparison of the exact and numerical solutions for $N=50$ of Example 5.1.

Example 5.2. We reach the Laguerre polynomial solution $u(x, t)$ in Eq. (3) by repeating the procedure in Sec. 2.

$$
\begin{aligned}
& \frac{\partial u(x, t)}{\partial t}=\frac{\partial^{2} u(x, t)}{\partial x^{2}}+2 \mathrm{e}^{-t}-\mathrm{e}^{\frac{t}{2}} u(x, t / 2) \\
& +\int_{0}^{t} u(x, \tau) \mathrm{d} \tau
\end{aligned}
$$

$u(x, 0)=x-x^{2}, u(0, t)=u(1, t)=0,0 \leq x t \leq 1$. The exact solution of the problem is $u(x, t)=\left(x-x^{2}\right) \mathrm{e}^{-t}$. Tables I-II show the comparison of absolute, $L_{2}, L_{\infty}$ and RMS errors for $N=40$ for Example 5.1 and Example 5.2 , respectively.

\section{Conclusion}

In this study, the approximate solutions of a class of parabolic type delay partial differential equations with some integral terms under the initial and boundary conditions have been presented. The technique we used is a new developed Laguerre collocation method and it can be applied to problems connected with hyperbolic and elliptic type delay partial differential equations with integral terms. Also, the method can be extended to nonlinear delay problems; but some modifications are required.
Comparison of the errors of Example 5.1.

TABLE I

\begin{tabular}{c|c|c|c|c}
\hline \hline$x, t$ & Absolute & \multicolumn{3}{|c}{ Error analysis } \\
\cline { 3 - 5 } & error & $L_{2}$ & $L_{\infty}$ & RMS \\
\hline 0.0 & $5.212 \mathrm{E}-08$ & $6.321 \mathrm{E}-09$ & $3.417 \mathrm{E}-09$ & $2.269 \mathrm{E}-09$ \\
0.2 & $2.653 \mathrm{E}-08$ & $2.120 \mathrm{E}-09$ & $9.854 \mathrm{E}-10$ & $9.110 \mathrm{E}-09$ \\
0.4 & $2.544 \mathrm{E}-09$ & $5.531 \mathrm{E}-10$ & $8.761 \mathrm{E}-10$ & $9.952 \mathrm{E}-10$ \\
0.6 & $4.159 \mathrm{E}-09$ & $9.204 \mathrm{E}-11$ & $7.029 \mathrm{E}-12$ & $5.217 \mathrm{E}-11$ \\
0.8 & $6.852 \mathrm{E}-09$ & $1.023 \mathrm{E}-10$ & $4.157 \mathrm{E}-12$ & $9.178 \mathrm{E}-10$ \\
1.0 & $3.258 \mathrm{E}-08$ & $2.859 \mathrm{E}-09$ & $3.114 \mathrm{E}-10$ & $7.145 \mathrm{E}-09$
\end{tabular}

Comparison of the errors of Example 5.2.

TABLE II

\begin{tabular}{c|c|c|c|c}
\hline \hline$x, t$ & Absolute & \multicolumn{3}{|c}{ Error analysis } \\
\cline { 3 - 5 } & error & $L_{2}$ & $L_{\infty}$ & RMS \\
\hline 0.0 & $3.586 \mathrm{E}-4$ & $8.613 \mathrm{E}-4$ & $4.562 \mathrm{E}-6$ & $5.426 \mathrm{E}-7$ \\
0.2 & $7.103 \mathrm{E}-4$ & $6.125 \mathrm{E}-5$ & $9.123 \mathrm{E}-8$ & $2.931 \mathrm{E}-9$ \\
0.4 & $2.503 \mathrm{E}-6$ & $6.297 \mathrm{E}-5$ & $1.953 \mathrm{E}-7$ & $9.513 \mathrm{E}-8$ \\
0.6 & $4.812 \mathrm{E}-4$ & $2.038 \mathrm{E}-4$ & $2.006 \mathrm{E}-7$ & $6.200 \mathrm{E}-7$ \\
0.8 & $8.216 \mathrm{E}-4$ & $9.256 \mathrm{E}-5$ & $7.630 \mathrm{E}-8$ & $2.230 \mathrm{E}-8$ \\
1.0 & $9.366 \mathrm{E}-4$ & $1.956 \mathrm{E}-4$ & $9.020 \mathrm{E}-6$ & $4.920 \mathrm{E}-7$
\end{tabular}

Hence, it would be very interesting to do research in this direction.

\section{Acknowledgments}

This work is founded by Manisa Celal Bayar University Department of Scientific Research Projects, with grant ref. $2014-151$

\section{References}

[1] J. Wu, Theory and Applications of Partial Functional Differential Equations, Springer, New York 2006.

[2] X. Fu, W. Zhang, J. Math. Anal. Appl. 191, 473 (1995).

[3] A. Tezel Özturan, Acta Phys. Pol. A 130, 14 (2016).

[4] A. Daamouche, D. Fares, I. Maalem, K. Zemmouri, Acta Phys. Pol. A 130, 28 (2016).

[5] B. Kırış, O. Bingöl, R. Şenol, A. Altıntaş, Acta Phys. Pol. A 130, 55 (2016).

[6] S. Kabashi, S. Bekteshi, S. Ahmetaj, B. Saramati, V. Veliu, Acta Phys. Pol. A 130, 122 (2016).

[7] N. El Houda Benmansour, M. Bouamoud, M. Sahlaoui, Acta Phys. Pol. A 130, 145 (2016).

[8] A. Marzocchi, E. Vuk, ZAMP 54, 224 (2003).

[9] B. Gürbüz, M. Sezer, Appl. Math. Comput. 242, 255 (2014).

[10] A. Özdemir, Z. Erdem, I. Usuman, Acta Phys. Pol. A 130, 228 (2016).

[11] M. Koru, O. Serçe, Acta Phys. Pol. A 130, 453 (2016).

[12] Ö. Karaçalı, Acta Phys. Pol. A 130, 249 (2016).

[13] B. Bülbül, M. Sezer, Math. Prob. Eng. 2013, 869749 (2013).

[14] B. Gürbüz, M. Sezer, Acta Phys. Pol. A 130, 194 (2016).

[15] F. Fakhar-Izadi, M. Dehghan, J. Comput. Appl. Math. 235, 4032 (2011). 